Voix et Images

volxetimages

\title{
Le roman historique : une littérature « nourrissante »?
}

\section{Allan Walsh}

Volume 13, numéro 3 (39), printemps 1988

Jack Kerouac et l’imaginaire québécois

URI : https://id.erudit.org/iderudit/200741ar

DOI : https://doi.org/10.7202/200741ar

Aller au sommaire du numéro

\section{Éditeur(s)}

Université du Québec à Montréal

\section{ISSN}

0318-9201 (imprimé)

1705-933X (numérique)

Découvrir la revue

\section{Citer cet article}

Walsh, A. (1988). Le roman historique : une littérature « nourrissante »? Voix et Images, 13(3), 499-503. https://doi.org/10.7202/200741ar d'utilisation que vous pouvez consulter en ligne.

https://apropos.erudit.org/fr/usagers/politique-dutilisation/ 


\section{Le roman historique: une littérature «nourrissante»?}

\section{par Allan Walsh, Université de Toronto}

Le roman historique a souvent été relégué hors de la culture lettrée et sa valeur littéraire a été mise en doute, même si ce genre participe au Québec d'une longue tradition qui remonte aux Anciens Canadiens. Mais depuis quelques années, le roman historique semble avoir trouvé un renouveau de vigueur et de popularité. Il ne fait aucun doute que le succès de romans tels la Chambre des dames en France et les Filles de Caleb au Québec ont beaucoup contribué à revaloriser une production que certains considèrent comme secondaire. Mais on se demande toujours si le roman historique possède une véritable valeur esthétique; s'agit-il d'une littérature «nourrissante» qui vaille la peine d'être lue et étudiée?

Question à la fois provocante et intéressante, mais à laquelle, malheureusement, on répond généralement par la négative. En soulevant la question du manque de littérarité qui entacherait le genre, on pointe aussi le problème de la marginalisation qui serait le propre du roman historique. Nous savons que le statut douteux de ce genre d'un point de vue institutionnel est dû en grande partie au fait qu'il est surtout associé aux débuts du réalisme en littérature. Loin de représenter une forme rudimentaire ou même dégradée du roman réaliste, nous croyons que le roman historique s'inscrit dans ce qu'on appelle maintenant la paralittérature. 
L'intérêt pour ce champ d'étude n'a cessé d'augmenter depuis une quinzaine d'années et l'on accepte maintenant le fait qu'une relation dialectique existe entre la paralittérature et ce qu'on appelait traditionnellement le domaine des Lettres. Le roman populaire devient alors un objet d'analyse culturel qui contribue, à part entière, à une compréhension de l'institution littéraire de notre société. La paralittérature que l'on avait abandonnée dans une zone d'inconscience culturelle peut commencer à parler d'elle-même comme le fait depuis un bon moment la «haute littérature». Le roman historique occupe une niche privilégiée à l'intérieur de ce champ d'enquête, car il met en scène non seulement des contenus fictionnels, mais aussi des contenus historiques. Les trois romans que nous présentons ici appartiennent à cette sous-catégorie et permettent d'entrevoir la variété des rapports qui peuvent exister entre littérature et histoire.

Louise Simard et Jean-Pierre Wilhelmy font revivre dans la Guerre des autres ${ }^{1}$ un épisode de notre histoire que les chercheurs et les historiens semblent avoir oublié: la participation sur le sol canadien de mercenaires allemands au moment de la Guerre d'indépendance américaine. Plus de 30000 recrues avaient été «louées» par des princes allemands aux autorités britanniques afin de repousser l'invasion des rebelles américains en territoire canadien. Le conflit dura sept ans, mais l'impact de la présence allemande fut de longue durée, car 2400 mercenaires décidèrent de rester au pays. Si là dramatisation de cette période s'inspire de faits historiques, empruntés en grande partie aux recherches de J.-P. Wilhelmy ${ }^{2}$, les personnages et les scènes que les auteurs représentent sont pour l'essentiel d'ordre fictif.

À travers tout le roman, un conflit entre deux personnages motive l'intrigue. Johann Vogel, un jeune étudiant de 23 ans, est arrêté pour ses opinions démocratiques par un militaire endurci, le lieutenant Georg Beyer. L'arrestation et l'enrôlement forcé ne sont que les premières difficultés que Johann affrontera. S'enchaînera une série d'actions et d'aventures qui amènera finalement Beyer et Vogel à Québec en 1776, où ils participeront aux campagnes militaires contre les rebelles américains. Tout, dans ce texte, rappelle le roman d'aventures ${ }^{3}$, car les personnages sont surtout définis à partir des difficultés et des obstacles qu'ils réussissent à surmonter en obéissant à un code d'honneur qui demeure sous-jacent au texte. Malgré un effort marqué pour leur donner une certaine profondeur sur le plan psychologique, un nombre restreint de traits physiques et psychologiques les caractérisent et les transformations personnelles se font plutôt rares. Il faut donc avouer que les personnages restent stéréotypés, sans toutefois tomber dans le ridicule.

Si l'évolution psychologique des personnages est sacrifiée, c'est parce que le but de l'œuvre est plutôt de décrire les conflits qui ont eu lieu entre les diverses cultures en contact en Amérique du Nord. Canadiens, Anglais, Allemands et Indiens occupent tous le même territoire et les affrontements abondent. Un des 
lieux privilégiés de ces conflits est la demeure de l'habitant canadien chez qui les mercenaires allemands sont billetés pendant l'hiver. Méfiance, jalousie et haine caractérisent les rapports entre les différents groupes. Ayant mis en place un certain nombre de contradictions, le texte nous offre une seule solution pour les résoudre: l'amour. L'intrigue amoureuse devient alors le moteur de la fiction et réussit à imposer sa loi au texte. La vie privée et les aventures des personnages dominent le roman et l'Histoire ne joue qu'un rôle secondaire et circonstanciel dans la construction de cette œuvre.

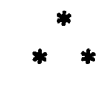

Si la Guerre des autres évolue autour d'une histoire d'amour, l'auteure de Le Moyne Picoté 4 , Agnès Guitard, fonde son roman sur un procès pour «rapt et séduction» intenté contre Pierre Le Moyne d'Iberville en 1686. Le procès durera à peu près 900 jours et l'auteure tient à nous en faire suivre l'évolution ainsi que l'impact et les retombées qu'il aura sur les personnes impliquées. Comme Simard et Wilhelmy, Guitard s'inspire de faits réels, mais sa vision du roman historique differe fondamentalement de celle de ces deux auteurs, car elle n'hésite pas à dépeindre d'authentiques personnages et à les mettre en scène dans de véritables épisodes historiques.

Guitard reconstitue des événements de notre histoire telle que l'expédition du chevalier de Troyes à la baie d'Hudson en 1686. Le rôle traditionnellement «héroïque» joué par d'Iberville dans ce genre de narration est modifié par l'auteure de différentes façons; on apprend par exemple — fait rarement consigné dans les livres d'histoire - qu'il s'est joint à l'expédition pour échapper à son procès. Le texte transforme ainsi un personnage souvent unidimensionnel et lui donne une forme humaine et complexe. À cette histoire «officielle», Guitard juxtapose le récit des gens oubliés par l'Histoire, plus précisément celui de la famille Picoté. On y découvre les sentiments de Jeanne Picoté à qui d'Iberville aurait fait un enfant. Le lecteur explore ses désirs et ses regrets, son amour et sa peur ainsi que l'isolement complet que lui imposent sa famille et la société de l'époque. On suit aussi les différentes démarches de sa sœur et tutrice Françoise, qui engage les procédures judiciaires contre d'Tberville. L'analyse des comportements et du caractère des personnages est efficace et loin d'être superficielle. L'auteure réussit à nous surprendre et ce, malgré le fait que les personnages se multiplient au fur et à mesure que le roman avance.

En fait, c'est à travers la juxtaposition de ces deux histoires (officielle et privée), et peut-être aussi par l'intermédiaire du mouvement entre elles, que le lecteur découvre que c'est toute l'histoire de la Nouvelle-France à la fin du $17 \mathrm{e}$ siècle qui se joue. L'enquête judiciaire portant sur une question de mours n'est que la toile de fond grâce à laquelle Guitard nous transporte au-delà des détails et nous fait participer à l'atmosphère sociale et politique de l'époque. Ainsi, la forme et le mouvement même du texte nous aident à découvrir progressivement l'Histoire. 
Si l'originalité d'Agnès Guitard consiste en la juxtaposition de deux récits pour faire de l'Histoire l'objet de son enquête, l'envergure du roman de Paul Ohl est bien différente. Katana 5 , son premier roman, nous emmène hors du Québec vers un monde à la fois exotique et violent: l'univers féodal japonais du $17 \mathrm{e}$ siècle. Pour nous communiquer sa vision du Japon d'autrefois, Paul Ohl choisit une stratégie narrative bien précise.

D'un côté, il met en scène l'histoire et les efforts du seigneur Tokugawa pour réunifier son pays divisé par l'influence des chrétiens venus d'Europe. On assiste à sa longue campagne militaire pour devenir le maître incontesté du Japon. Les affrontements et les combats qu'il livra au début du $17 \mathrm{e}$ siècle sont à contempler par le lecteur comme s'il s'agissait d'un gigantesque spectacle à la fois atroce, absurde et attirant où se mêlent beauté et violence barbares.

Parallèlement au texte historique, l'auteur tisse un autre récit, fictif celui-là, dans lequel nous rencontrons Kikusui, un enfant trouvé qui a passé toute sa vie dans un monastère. Le début du roman coïncide avec le moment où son maître le convoque pour lui parler de son passé. Ne pouvant plus se dérober à ses origines de samouraï, il est contraint de quitter le monastère pour aller courir le monde afin de s'initier à la vie et faire l'apprentissage de la voie du sabre (katana en japonais). Nous suivrons le cheminement initiatique de ce sympathique personnage et découvrirons avec lui, entre autres, les secrets de l'art guerrier ainsi que ceux de la cérémonie du thé et de l'arrangement floral japonais. Ayant juxtaposé l'Histoire et la fiction, Paul Ohl se permet d'entremêler les deux récits. Le sort de Kikusui est alors inévitablement lié à celui du seigneur Tokugawa pour lequel il devient un samouraï.

Un aspect intéressant de ce roman vient du fait que les deux récits progressent côte à côte sans qu'aucun ne l'emporte sur l'autre; situation fort différente de ce que nous avons repéré dans nos deux premiers textes où le romanesque ou l'Histoire a dominé. Ainsi, chacun de ces romans a articulé de façon individuelle ses contenus historique et fictionnel, ce qui nous porte à croire que trois formes distinctes du roman historique sont alors possibles.

En somme, la lecture de ces trois romans montre la diversité qui peut exister à l'intérieur d'un seul genre. À première vue, la structure du roman historique semble assez traditionnelle, mais ce genre se permet aussi une variété surprenante de thèmes, de styles et de contenus référentiels. Certains textes comme la Guerre des autres prennent peu de risques et s'insèrent dans la tradition du roman d'aventures. D'autres tels Katana et surtout Le Moyne Picoté font preuve non seulement d'une écriture originale et d'une narration complexe, mais aussi d'un certain nombre de jeux formels. Cependant, ce n'est qu'en poursuivant les recherches qui s'imposent que nous pourrons vraiment comprendre la place qu'occupe le roman historique à l'intérieur de l'institution littéraire de notre société. 
1 Montréal, La Presse, 1987, 408 p.

2 Les Mercenalres allemands au Québec du XVIIIe siècle et leur apport à la population, Québec, Maison des mots, 1984, 332 p.

3 Nous empruntons dans les pages qui suivent la définition du roman d'aventures que François Gallays a présentée dans son article «Faut-il brûler Clavel?», Lettres québécolses, $n^{\circ} 47$, automne 1987, p. 27-28.

4 Le Moyne Plcoté. Un procès pour rapt et séduction contre Plerre Le Moyne d'Iberville, Montréal, Québec/Amérique, 1987, 472 p.

5 Katana. Le roman du Japon, Montréal, Québec/Amérique, 1987, 528 p. 